\title{
Load Shift Protocol Design in ATM-Based VOD Systems*
}

\author{
Jean-Lien C. Wu, Jen-Kai Chen, Hung-Wen Chao \\ Department of Electronic Engineering \\ National Taiwan University of Science and Technology \\ 43, Keelung Road, Section 4, Taipei, Taiwan, R.O.C. 106 \\ Email: jcw@nlhyper.et.ntust.edu.tw
}

\begin{abstract}
Load shift is a basic operation for load balancing algorithms. In this paper, we study the load shift problem in an ATM-based distributed video-on-demand (VOD) system. Two different system configurations are considered for the load shift. One allows more than one video streams being active simultaneously from multiple video servers to a set-top box (STB), in which no-gap load shift is possible. The other one allows only one video stream between a STB and the VOD system. With respect to this case, two transport schemes are proposed: dynamic rate control with load shift (DRCLS) and constant rate control with load shift (CRCLS). For DRCLS, new water lines of the STB buffer have to be specified for flow control in cases of load shifts. For CRCLS, we compute the new feasible parameter set, which includes the required bandwidth, the minimal STB buffer size and the pre-download time. Finally, we compare the differences between CRCLS and DRCLS.
\end{abstract}

\section{Introduction}

In recent years, the progress of data compression technology makes the multimedia application becoming more and more popular. Furthermore, the continuous progress of computer network technology speeds the development of Video-on-Demand (VOD) technologies and makes the implementation of VOD services easier. However, in a distributed VOD system, if static load assignment is used, unbalanced load conditions are likely to occur due to non-uniform traffic loads among VOD servers. To improve system throughput, dynamic load balancing algorithms [1] can be applied, in which load shift is the basic operation.

With the example [1], shown in Fig. 1, there are two video servers, namely $\mathrm{A}$ and $\mathrm{B}$, which contains duplicated

\footnotetext{
* This research was supported by National Science Council of the Republic of China under grant NSC 86-2213-E-011-062.
}

copies of several films, according to a placement policy. Suppose that films 1, 2, 3 and 4 are placed in video server A, which is feeding film 3 for one of the users and is in a fully loaded state. In other words, all video channels of the video server A are occupied. Films 1, 2 and 3 are stored in video server B where there still are available channels. If a user issues a request for film 4, it can not get service from video server A because of no available channel in A. However, this system is capable of serving this new request if the following steps are performed : (1) the load for film 3 is shifted from video server A to video server B; (2) the released channel in A is assigned to serve it with film 4. By this way, we can accommodate more active users simultaneously in heavy load situations.

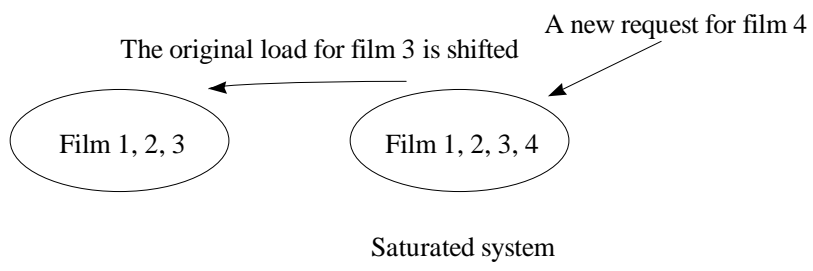

Video Server B Video Server A

Fig. 1 Increasing service capacity by load shift

To achieve on-the-fly load balance, load shift is the basic operation that could be performed at any playback point of a video stream. But it will introduce temporary idle in data transmission that has an impact upon the transport scheme. That is the main topic investigated in this paper. In section 2, two load shift protocols are proposed for different system configurations. For the with-gap load shift protocol, we have to modify the transport schemes to guarantee continuous real-time display while load shifts are performed. In section 3, two transport schemes are proposed for load shift: dynamic rate control with load shift (DRCLS) that is a variant of the water-level transport protocol [2], and constant rate control with load shift (CRCLS) that is a variant of the constant-rate transmission and transport protocol[3]. Simulation results and discussions are shown in section 4 . 
The conclusion is in Section 5.

\section{Load Shift Protocols for Different System Architectures}

In this section, two transmission protocols are proposed to support load shift for different system architectures. One is called the no-gap protocol for the pure ATM (PATM) architecture. The other is called the with-gap protocol for the intermediate node architecture.

\subsection{PATM VOD System Architecture}

The PATM VOD system architecture implies that both the video servers and the STBs are directly connected to the ATM network, as shown in Fig. 2. Users can have direct access to different video servers with multiple switched virtual circuits (SVCs) simultaneously, i.e. STBs have enough bandwidth to get concurrent video service.

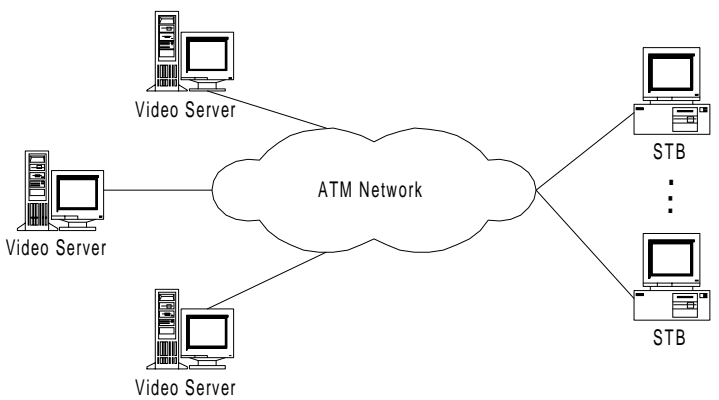

Fig. 2 The PATM VOD system architecture

In order to reduce the response time of load shift operation, both the original video server and the successive video server are allowed to transmit data concurrently to the same STB. Fig. 3. depicts the no-gap load shift scenario, described as follows.

step 1: The resource manager sends a control signal to inform the original video server (named video server 1) to move the load on a certain video stream to a specific successive video server (named video server 2).

step 2: Video server 1 sets up a signaling SVC to the successive video server.

step 3: Video server 1 sends a notification message to the STB to indicate the address of the successive video server.

step 4: The STB sets up an SVC to video server 2 for carrying the successive video stream after receiving the notification message from video server 1 .

step 5: STB replies an ACK message to video server 1 while the video stream SVC has been set up.

step 6: Video server 1 determines the seem point and informs the STB and video server 2. Then it continues data transmission until reaching the seem point, before that the successive video stream will have started.

step 7: Video server 2 begins to transmit the video starting from the seam point.

step 8: After completion of the transmission at the seam point, video server 1 tears down the data and signal connections with the STB and the signaling connection with the video server 2 .

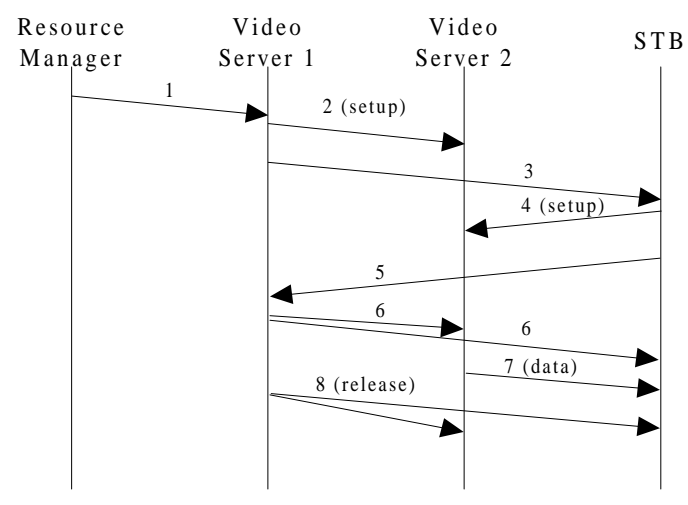

Fig. 3. Scenario of the no-gap protocol

\subsection{Intermediate Node VOD System Architecture}

The intermediate node architecture is shown in Fig. 4 and described as follows. The STB and the video servers are connected to the access network and ATM WAN respectively. The STB has access to the video server through the access network, the intermediate node, and the ATM WAN.

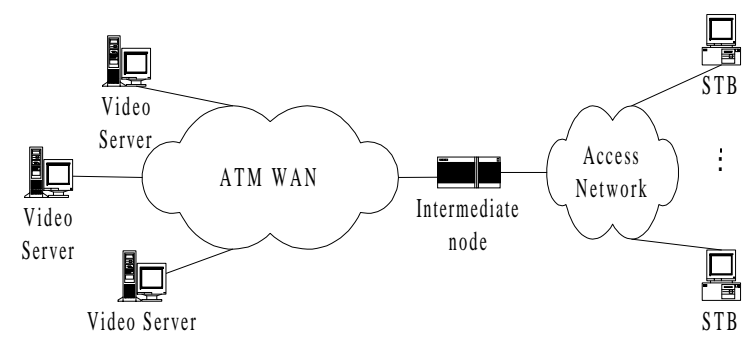

Fig. 4. Intermediate node VOD system architecture

Because the resources (bandwidth or buffers) in the access network, the intermediate node, or the backbone network may not be enough to provide the two video streams to the STB simultaneously, only one downstream virtual circuit is allowed between a STB and the VOD system while load shift is performed. For example, the downstream of ADSL-1 can accommodate only one 1.5 Mbps MPEG-1 video stream. Hence, it is impossible to 
have two video servers transmitting video data simultaneously to the STB without buffering in the intermediate node. In another point, if the bandwidth of the intermediate node are not enough to set up another additional channel, the above case will still hold even the intermediate node has enough buffer for the successive video stream. In such situations, the no-gap control scheme does not work. So, we need to design transport schemes for the with-gap case, in which only one video stream can exists between the STB and the distributed VOD system.

We explore a scenario of the with-gap protocol in the following steps, depicted in Fig. 5.

step 1: The resource manager sends a control signal to inform the original video server (named video server 1) to move the load on a certain video stream to a specific successive video server (named video server 2 ).

step 2: Video server 1 sends a notification message to the STB to indicate the seam point and the address of the successive video server.

step 3: The STB releases the data and signaling connections with video server 1 .

step 4: The STB sets up the data and signaling connections with video server 2 .

step 5: The STB sends a notification point to video server 2 to indicate starting transmission from the seam point.

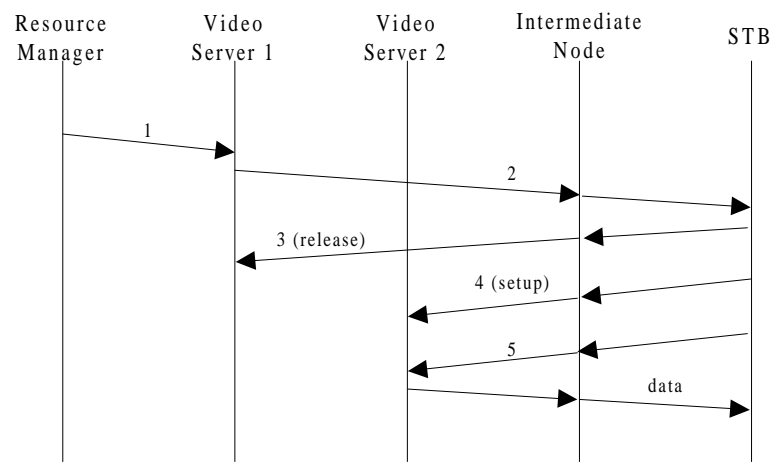

Fig. 5 An example of CRCLS with-gap protocol

According to the with-gap protocol, the delays caused by the following operations are no longer negligible.

(1) release time of the original SVC,

(2) setup time of the successive SVC,

(3) round-trip delay of the notification signaling data,

(4) processing time of the notification signaling data,

(5) disk access delay of the successive frame starting at the seam point, and

(6) transmission time of the first frame sent by the successive video server.

The dominant factors are the setup and release time of
SVCs. The rest cost only a few tens of milliseconds totally. A reasonable assumption about the setup and release time is to be a random number in the range of hundreds of milliseconds to a few seconds in an ATM WAN. For example, if the estimated worst-case gap interval is one second, the client has to cache at least $150 \mathrm{~KB}$ in its video buffer for a $1.2 \mathrm{Mbps}$ video stream to prevent starvation while a load shift takes place.

\section{Transport Protocols for Load Shift}

In this section, two transport protocols for the with-gap cases are proposed for video transmission while on-the-fly load shift is possible. The former is called the dynamic rate control with load shift (DRCLS) protocol that is a variant of the water-level transport protocol [2], and the latter is called the constant rate control with load shift (CRCLS) protocol that is a variant of the constant-rate transmission and transport protocol[3].

\subsection{Dynamic Rate Control with Load Shift}

To provide load shift using dynamic rate control [2], we label the STB buffers by waterlines as thresholds, used in load shift control, as shown in Fig. 6. Overflow control still uses the high water line that is proposed in [2] and not depicted in Fig. 6. Region IV resides in between the bottom of the buffer and the water line Original_LW. If the STB buffer is in this region, load shift requests will not be accepted. Region III resides in between water line Original_LW and water line No_Data_Recv. The region from the bottom to No_Data_Recv is used to support the data consumption during the gap interval of load shift. Region II is between water line No_Data_Recv and water line Load_Shift_LW. The highness of the region II is equal to that of Region IV. Region I is between the top of the buffer and water line Load_Shift_LW. In this region, the STB has enough buffered data to handle load shift without starvation.

The scenario of DRCLS is described as follows. First, we needs to check the position of the water line of the STB buffer at the instant when the original video server has completed the setup of an SVC to the successive video server. If the water line is in region I, the contiguous actions for load shift can be executed directly without changing transmission rate because there are enough frames in the STB buffer to overcome the load-shift gap. If the water line is in region II, the contiguous actions for load shift can be executed, but the STB should inquire a higher transmission rate to avoid the starvation after load shift.

If the water line is in region III, the STB will send a 
control message to the original video server to ask for a higher transmission rate, as shown in step (i) of Fig. 7. After the water line rises up to region II, the STB should send a control message to the original video server to stop the transmission and inquire the seam point, as shown in step (ii). Then, the original video server notifies the STB the seam point, as shown in step (iii), and the follow-up actions are executed as described as the case that the water line is in region II.

If the water line is in region IV, that is, under water line Original_LW. Because the DRCLS is an enhancement of the general dynamic rate control[2], the speedup control signal will be issued when the water line of STB buffer is below Orignal_LW. Therefore, load shift has to wait until the water line reaches No_Data_Recv.

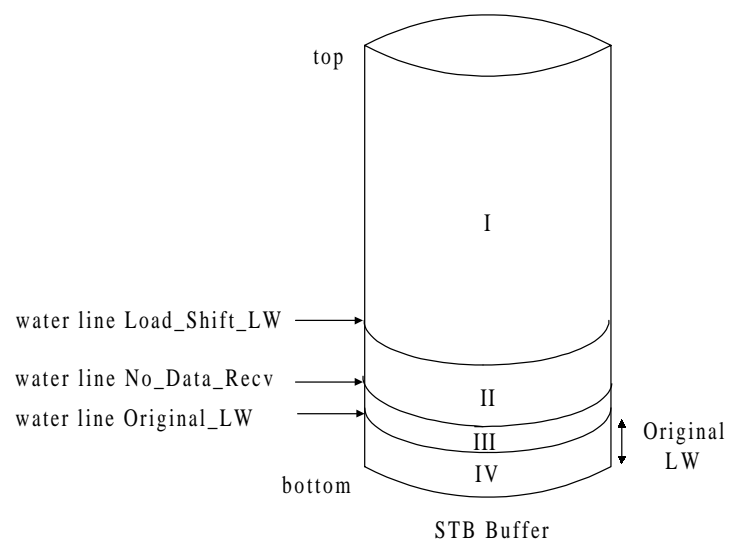

Fig. 6 Detailed configuration of the STB buffer

\subsection{Constant Rate Control with Load Shift}

Based on different conditions that generate the load shift, we consider the cases of Absolute Load Shift (ALS) and Semi Load Shift (SLS). ALS indicates that load shift will be executed exactly once during playback. SLS indicates that load shift is executed at most once during playback. Both of them are compared with the case of No Load Shift (NLS) indicating that no load shift is performed during playback. Although ALS is not realistic for load shift is executed only when it is held useful for load balance, we use it to discuss the SLS scheme. If the maximum number of load shifts during playback is $m$, the SLS scheme can be used for generalization.

Let $b_{\text {min }}$ denotes the minimum feasible transmission rate which guarantees no starvation during playback, and $b_{\max }$ denotes the maximum feasible transmission rate which guarantees no buffer overflow during playback. With the algorithm proposed in [3], $b_{\min }$ and $b_{\max }$ can be computed in $O(N)$ time, given the pre-download time $d$ and the STB buffer size $B$. The minimum STB buffer size $B^{*}$ and its corresponding optimal transmission and pre- download time can be calculated in $O\left(N^{2} \log N\right)$ time. By reserving the necessary buffer space for load shift, we can calculate the feasible rate range for ALS. Then, the feasible rate range for SLS can be derived simply by intersecting the two feasible rate ranges of NLS and ALS, as shown in Fig. 8.

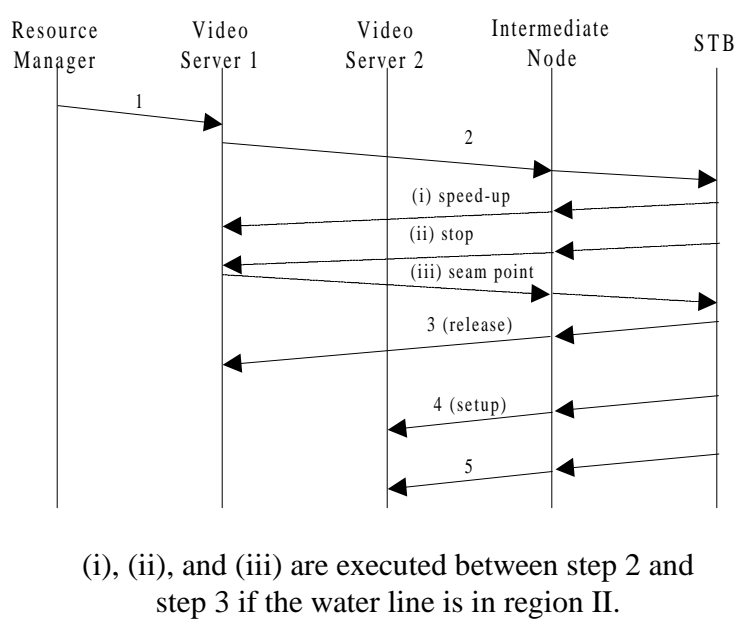

Fig. 7 Scenario of the DRCLS protocol

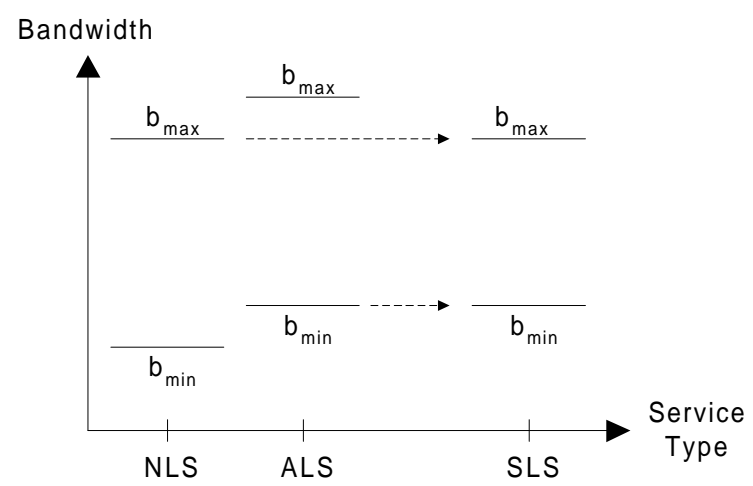

Fig. 8 Bandwidth range for SLS

\section{Simulation Results and Discussion}

In our experiment, we considered that there are two ATM switches interconnected via PVC and using Fore's Simple Protocol as the ATM NNI signalling protocol; the workstations with IP address A and B are attached to the port 1 of the Switch 1 and the port 3 of the Switch 2 respectively. Therefore, their ATM addresses are $(S=1$, $\mathrm{P}=1)$ and $(\mathrm{S}=2, \mathrm{P}=3)$ respectively.

\subsection{Cache of the ATM ARP}

The procedures of using Fore's APIs for video 
transmission in the server side are as follows: (1) atm_open, (2) atm_bind, (3) atm_gethostbyname, (4) atm_connect, (5) atm_send, and (6) atm_close. The procedures in the client side are as follows: (1) atm_open, (2) atm_bind, (3) atm_listen, (4) atm_accept, (5) atm_rec, and (6) atm_close.

The average setup time of an SVC is about $25 \mathrm{~ms}$ in our system configuration. Note that it is the best case because there are not any other traffic load in our ATM network. In order to diminish the gap interval of load shift, we adopt the cache mechanism for the IP to ATM address mapping during the SVC setting up such that the average setup time is reduced to about $8 \mathrm{~ms}$, improving about $17 \mathrm{~ms}$.

Because load shift is performed only in the more heavily loaded situations, the setup time of an SVC could be up to a few hundreds milliseconds in a reasonable guess. In the following comparisons, we assume the gap interval of load shift is $500 \mathrm{~ms}$ with the SLS, not using cache mechanism. In the SLS plus cache mechanism, the gap interval is $483 \mathrm{~ms}$. The transmission delay of data packet is $86.4 \mathrm{~ms}$ and the transmission delay of control packets is $0.1 \mathrm{~ms}$ in our scenario.

\subsection{Different Services and Buffer Sizes for CRCLS}

For the constant rate control, we consider four service types, i.e. NLS, ALS, SLS and Semi-Load Shift with Cache (SLSC), to compute the minimal STB buffer requirement for the film "Star War"[6].

We assumed that 47 bytes of payload are used to transport the MPEG frames. Besides, the Start War consists of 174136 frames totally and 24 frames/sec rate. The average number of cells per frame is 41.99 . There are 493.28 cells for the peak frame and 2.04 cells for the minimal frame.

The results for different service types of load shift are shown in Table 1. We also list the percentages of increasing in terms of the minimal STB buffer size, network bandwidth, the number of pre-download frames, and the pre-download time due to load shift.

For a larger STB buffer size, there are more $(b, d)$ pairs available, where $b$ is the feasible transmission bandwidth of an ATM virtual channel (VC) per second in unit of cells and $d$ is the pre-download time. Therefore, there are more choices that network resource manager can properly assign according to the load of system at that time. Our results show that increasing $10 \%$ in buffer size will decrease the minimum feasible bandwidth from 991.972 cells/s to 974.961 cells/s. So, the tradeoff between bandwidth effective utilization, STB buffer cost, and predownloaded time can be determined by the resource manager according to the system load at that time.

\begin{tabular}{|c|c|c|c|c|}
\hline & $\begin{array}{c}\text { Min } \\
\text { STB } \\
\text { Buffer } \\
\text { Size } \\
\text { (cells) }\end{array}$ & $\begin{array}{c}\text { Band- } \\
\text { width } \\
\text { (cells/s) }\end{array}$ & $\begin{array}{c}\text { Pre- } \\
\text { download } \\
\text { Size } \\
\text { (frames) }\end{array}$ & $\begin{array}{c}\text { Pre- } \\
\text { download } \\
\text { time (sec.) }\end{array}$ \\
\hline NLS & 473890 & 995.185 & 1163 & 37.18 \\
\hline ALS & 473887 & 995.186 & 1175 & 37.67 \\
\hline SLS & 474834 & 995.186 & 1175 & 37.67 \\
\hline SLSC & 474374 & 995.183 & 1175 & 37.6701 \\
\hline \hline $\begin{array}{c}\text { (SLS- } \\
\text { NLS)/NLS }\end{array}$ & $0.2 \%$ & $0 \%$ & $1.03 \%$ & $1.32 \%$ \\
\hline $\begin{array}{c}\text { (SLSC- } \\
\text { NLS)/NLS }\end{array}$ & $0.1 \%$ & $0 \%$ & $1.03 \%$ & $1.3206 \%$ \\
\hline
\end{tabular}

Table 1. Comparison of different services under the CRCLS

\subsection{DRCLS vs. CRCLS}

In Table 2, We compare the above two rate control schemes with load shift in the terms of the minimal STB buffer size, bandwidth of network, system response time, the number of pre-download frames and pre-download time.

In summary, there are many advantages by using CRCLS, including that the management and implementation are easier, quick system response time, and the requirement of smaller network bandwidth. The advantages of DRCLS include smaller minimal STB buffer size, smaller number of pre-downloaded frames and no pre-download waiting time.

\begin{tabular}{|c|c|c|c|c|c|}
\hline & $\begin{array}{c}\text { Min STB } \\
\text { Buffer } \\
\text { Size } \\
\text { (cells) }\end{array}$ & $\begin{array}{c}\text { Network } \\
\text { Band- } \\
\text { width } \\
\text { (cells/sec) }\end{array}$ & $\begin{array}{c}\text { Pre- } \\
\text { download } \\
\text { Size and } \\
\text { Time } \\
\text { (frames/sec) }\end{array}$ & $\begin{array}{c}\text { System } \\
\text { Response } \\
\text { Time }\end{array}$ & $\begin{array}{c}\text { Manage- } \\
\text { ment and } \\
\text { Implemen- } \\
\text { tation }\end{array}$ \\
\hline CRCLS & 474834 & 995.186 & $1175 / 37.67$ & short & easy \\
\hline DRCLS & 43484 & $\begin{array}{c}\text { Hi-Rate: } \\
11839 \\
\text { Lo-Rate: } \\
49\end{array}$ & $0 / 0$ & $\geq$ CRCLS & more \\
complex
\end{tabular}

Table. 2 Comparisons of CRCLS and DRCLS

\section{Conclusion}

In this paper, in order to provide the load shift service in ATM-based distributed VOD system, we proposed a load shift protocol for the no-gap case and two revised transport protocol for the with-gap case, i.e. the DRCLS protocol and the CRCLS protocol. 
For CRCLS, the calculation of transmission parameters for load shift were given. The tradeoff between the buffer size of the STB and the required transmission bandwidth is explored. In the scenario of Star War, increasing 1/10 buffer size will decrease the required bandwidth from 991.972 cells/s to 974.961 cells/s because there are more $(b, d)$ pairs available for larger STB buffer size. Secondly, the comparisons for NLS, ALS, SLS and SLSC are explored. The comparison results show that increasing $0.2 \%$ STB buffer size will obtain the load shift service in the scenario of Star War. Finally, we also compare the differences between the DRCLS and CRCLS. Although CRCLS needs more STB buffer size to smooth the jitter characteristic of VBR-encoded film, it is easier in implementation.

Our future work is to find efficient transmission planning algorithms for the normal video transmission and the load shift case.

\section{Acknowledgment}

The authors thank M. Garrett for making the Star Wars trace available.

\section{References}

[1] J. L. Wolf, P. S. Yu, and H. Shachai, "DASD Dancing: A Disk Load Balancing Optimization Scheme for Video-onDemand Computer Systems," Proceedings of ACM SIGMETRICS, pp. 157-166, 1995.

[2] C. C. Yang, and J. H. Huang, "On the Design of a Transport Protocol for the Video-on-Demand System," Proceedings of ICOIN-10, Kyung-Ju, Korea, pp285-291, Jan. 1996.

[3] J. M. McManus and K. W. Ross, "Video-on-Demand Over ATM: Constant-Rate Transmission and Transport," IEEE JSAC, Vol. 14, No. 6, pp. 1087-1098, Aug. 1996.

[4] J. M. D. Rosario and G. Fox, "Constant Bit Rate Network Transmission of Variable Bit Rate Continuous Media in Video-On-Demand Servers," Multimedia Tools and Applications, Vol 2, No. 3, pp. 215-232, May 1996.

[5] J. Ni, T. Yang and D. H. K. Tsang, " CBR Transportation of VBR MPEG-2 Video Traffic for Video-On-Demand in ATM Networks," Proceeding of ICC'96, Dallas, TX, USA, Vol. 3, pp. 1391-1395, June 1996.

[6] MPEG.data Trace available via anonymous FTP from thumper.bellcore.com. 